\title{
Влияние разориентации подложки и ее предварительного травления на структурные и оптические свойства интегрированных гетероструктур GaAs/Si(100), полученных методом газофазной эпитаксии
}

\author{
() П.В. Середин ${ }^{1}$, Д.Л. Голощапов ${ }^{1}$, Д.С. Золотухин ${ }^{1}$, А.С. Леньшин ${ }^{1}$, А.Н. Лукин ${ }^{1}$, \\ Ю.Ю. Худяков ${ }^{1}$, И.Н. Арсентьев ${ }^{2}$, А.В. Жаботинский ${ }^{2}$, Д.Н. Николаев ${ }^{2}$, Н.А. Пихтин ${ }^{2}$ \\ ${ }^{1}$ Воронежский государственный университет, \\ 394006 Воронеж, Россия \\ ${ }^{2}$ Физико-технический институт им. А.Ф. Иофрфе Российской академии наук, \\ 194021 Санкт-Петербург, Россия \\ E-mail: paul@phys.vsu.ru
}

(Получена 27 сентября 2017 г. Принята к печати 10 октября 2017 г.)

\begin{abstract}
Впервые удалось показать, что управление структурными и оптическими функциональными характеристиками интегрированных гетроструктур $\mathrm{GaAs} / \mathrm{Si}(100)$ может быть достигнуто за счет использования разориентированных подложек $\mathrm{Si}(100)$ и их предварительного травления. Рост эпитаксиального слоя $\mathrm{GaAs}$ на подложках кремния без образования антифазных доменов может быть выполнен на подложке, отклоненной от сингулярной плоскости (100) менее чем на $4-6^{\circ}$ или без использования переходного слоя из наностолбиков GaAs. Выполненная предварительная обработка кремниевой подложки в виде травления позволяет получить на ней методом газофазной эпитаксии пленку GaAs в монокристаллическом состоянии со значительно меньшим коэффициентом релаксации, что положительно отражается на ее структурном качестве. Эти данные находятся в хорошем согласовании с результатами ИК-спектроскопии на отражение, фотолюминесцентной и УФ-спектроскопии. Особенности оптических свойств интегрированных гетероструктур $\mathrm{GaAs} / \mathrm{Si}(100)$ в инфракрасной и ультрафиолетовой областях спектра также определяются значением коэффициента релаксации.
\end{abstract}

DOI: 10.21883/FTP.2018.08.46213.8737

\section{1. Введение}

Основным барьером в увеличении темпов развития оптоэлектронной промышленности, а также солнечной фотоэнергетики является относительно высокая себестоимость конечного продукта, поскольку основными материалами для производства такого рода высокоэффективных устройств являются полупроводники группы GaAs $[1,2]$. Хорошо известно, что технология производства $\mathrm{A}^{\mathrm{III}} \mathrm{B}^{\mathrm{V}}$ компонент и устройств далеко не дешева, а созданные на основе $\mathrm{A}^{\mathrm{III}} \mathrm{B}^{\mathrm{V}}$ приборы и устройства потребляют больше энергии, чем их кремниевые аналоги.

Поэтому наиболее перспективным подходом для решения вышеописанной проблемы является интеграция полупроводниковых соединений GaAs-группы с кремнием [3]. Появление технологий формирования на поверхности кремниевого чипа $\mathrm{A}^{\mathrm{III}} \mathrm{B}^{\mathrm{V}}$ функциональных элементов создаст предпосылки для появления новых оптоэлектронных устройств с принципиально новой архитектурой по сравнению с существующими комплементарными структурами металл-оксид-полупроводник (КМОП) [4].

Тем не менее интеграция $\mathrm{A}^{\mathrm{III}} \mathrm{B}^{\mathrm{V}}$ материалов с $\mathrm{Si}$ несет в себе целый ряд трудностей, главным образом обусловленных как несоответствием параметров кристаллической решетки гетеропары $\mathrm{A}^{\mathrm{III}} \mathrm{B}^{\mathrm{V}} / \mathrm{Si}$, так и разнородностью свойств кремния и полупроводниковых соединений $\mathrm{A}^{\mathrm{III}} \mathrm{B}^{\mathrm{V}}$ (к примеру, коэффициентов термического расширения, полярности материала, зонной структуры и т.д.) [1,5].

Для решения описанных проблем в настоящее время отработаны и изучены лишь некоторые способы и подходы, позволяющие совместить полупроводниковые материалы GaAs-группы и кремниевый чип [6], причем возникающие при этом трудности до сих пор преодолеваются ведущими исследовательскими группами с переменным успехом.

Так, в частности рост полярного полупроводника на неполярной подложке, приводящий к образованию антифазных доменов большой плотности, разрешается за счет применения подложек $\mathrm{Si}$ или $\mathrm{Ge}$, отклоненных от сингулярной плоскости (001) на 4-6 ${ }^{\circ}[5,7]$, или более затратного метода роста на переходном слое из наностолбиков GaAs [8]. Высокая ( 4\%) разница параметров кристаллических решеток для системы $\mathrm{GaAs} / \mathrm{Si}$, a также значимая разница в коэффициентах термического расширения способствуют образованию большого количества дислокаций и появлению микротрещин в пленке GaAs в процессе ее роста [6]. Данная проблема решается путем высокотемпературной обработки гетероструктур $\mathrm{A}^{\mathrm{III}} \mathrm{B}^{\mathrm{V}} / \mathrm{Si}$ (отжиги, термоциклирование), существенно улучшающих кристаллическое совершенство этих платформ, что в свою очередь неприемлемо при выращивании GaAs в окнах подложек $\mathrm{Si}$ с готовыми 
элементами интегральных схем, а также путем роста серий переходных буферных слоев $\mathrm{GaAs} / \mathrm{Ge} / \mathrm{Si}[9,10]$, что в свою очередь не всегда приемлемо.

Весьма интересным и перспективным для интеграции $\mathrm{A}^{\mathrm{III}} \mathrm{B}^{\mathrm{V}}$-материалов с $\mathrm{Si}$ нам кажется подход по созданию „податливых“ подложек на основе пористого слоя, сформированного непосредственно на $\mathrm{Si}$. И хотя известные единичные попытки роста на пористом слое показали перспективность такого подхода к формированию интегрированных $\mathrm{A}^{\mathrm{III}} \mathrm{B}^{\mathrm{V}} / \mathrm{Si}$, фактически это не привело к развитию предложенной технологии, что, на наш взгляд, связано с формированием слоя макро- и мезопористого кремния в роли податливой подложки $[11,12]$.

Следует отметить, что в литературе отсутствует информация о структурных и оптических свойствах GaAs, выращенного методом газофазной эпитаксии (ГФЭ) на „податливой“ подложке - тонком пористом слое $\mathrm{Si}$. Таким образом, цель нашей работы состоит в исследовании влияния степени разориентации подложки и ее предварительного травления на структурные и оптические свойства интегрированных ГФЭ гетероструктур $\mathrm{GaAs} / \mathrm{Si}(100)$.

\section{2. Материалы и методы}

Серия тестовых образцов была выращена на установке ГФЭ эпитаксии „ЕMCORE GS 3/100“ в вертикальном реакторе с высокой скоростью вращения подложкодержателя. Эпитаксиальная пленка каждого образца имела единую структуру, представленную на рис. 1. В качестве подложек были использованы пластины следующих соединений (см. табл. 1). Температура подложкодержателя во время эпитаксиального роста $700^{\circ} \mathrm{C}$, давление в реакторе 77 Торр, скорость вращения подложкодержателя 1000 об/мин. В качестве исходных реагентов использовались $\mathrm{Ga}\left(\mathrm{CH}_{3}\right)_{3}, \mathrm{Al}\left(\mathrm{CH}_{3}\right)_{3}$ и $\mathrm{AsH}_{3}$. Образцы выращивались в одном технологическом процессе. Скорость роста GaAs составила $\sim 770 \AA$ Аинн.

Пористый (нано) слой на поверхности Si (образец \#3) был сформирован путем травления в известном составе раствора: 1 часть $\mathrm{HF}+1$ часть уксусной кислоты +40 частей азотной кислоты в течение 1 мин.

Микроскопические исследования качества гетероинтерфейсов были выполнены на электронном микроскопе Libra 120 Carl Zeiss.

Таблица 1. Характеристики подложек, использованных для роста гетероструктур

\begin{tabular}{c|c|l}
\hline Образец & Тип подложки & \multicolumn{1}{|c}{ Характеристики } \\
\hline$\# 2$ & $\mathrm{GaAs}(100)$ & Полуизолятор \\
$\# 3$ & $\mathrm{Si}(100)$ & Разориентация $\sim 3^{\circ}$ \\
& & к $[110]+$ травление \\
$\# 4$ & $\mathrm{Si}(100)$ & Разориентация $\sim 3^{\circ}$ к $[110]$ \\
$\# 5$ & $\mathrm{Si}(100)$ & Разориентация $\sim 7.5^{\circ}$ к $[110]$
\end{tabular}

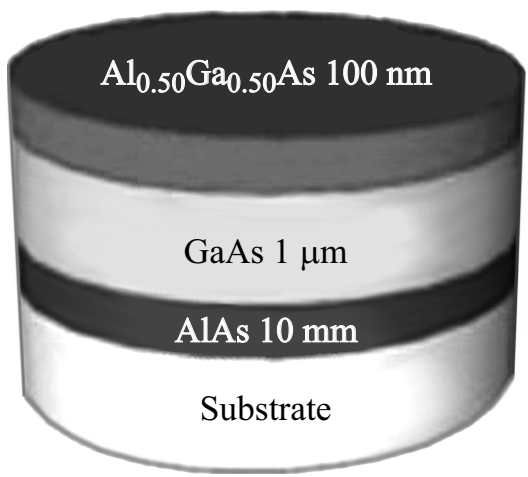

Рис. 1. Дизайн эпитаксиальных гетероструктур исследованных в работе образцов.

Рентгенофазовый анализ полученных образцов проводили методами рентгеновской дифракции, с использованием дифрактометра ДРОН 4-07 с кобальтовой трубкой $\mathrm{Co} K_{\alpha}=1.790 \AA$.

Структурное качество образцов и определение параметров решеток твердых растворов проводили методом рентгеновской дифракции на дифрактометре Seifert 3003 HR с четырех кружным гониометром и монохроматизированным излучением меди с длиной волны $\mathrm{Cu} K_{\alpha_{1}}=1.5405 \AA$.

Спектры фотолюминесценции гетероструктур получены при комнатной температуре от поверхности образцов по стандартной методике на основе монохроматора TRIAX550, охлаждаемого жидким азотом CCD-детектора при возбуждении аргоновым лазером с длиной волны 514.5 нм. Для фокусировки на поверхности использовался $10 \times$ объектив. Интенсивность лазерного излучения при возбуждении фотолюминесценции составляла $\sim 5 \mathrm{MBT} / \mathrm{cm}^{2}$.

Оптические свойства образцов изучались в диапазоне 190-900 нм, методом ультрафиолетовой (УФ) спектроскопии, с помощью прибора LAMBDA 650 фирмы Perkin Elmer, оснащенного универсальной приставкой URA, позволяющей получать спектры отражения в интервале углов падения от 8 до $80^{\circ}$. При этом рабочая схема приставки позволяет получать абсолютное отражение. Спектры отражения нами были получены при угле падения $67^{\circ}$.

Инфракрасные спектры отражения от гетероструктур были получены с использованием ИК фурье-спектрометра Vertex-70 Bruker.

\section{3. Экспериментальные результаты и их обсуждение}

\section{1. Структурные исследования}

На рис. 2 представлены обзорные дифрактограммы исследованных в работе образцов. Как видно из полученных данных, на дифрактограмме образца \#2 


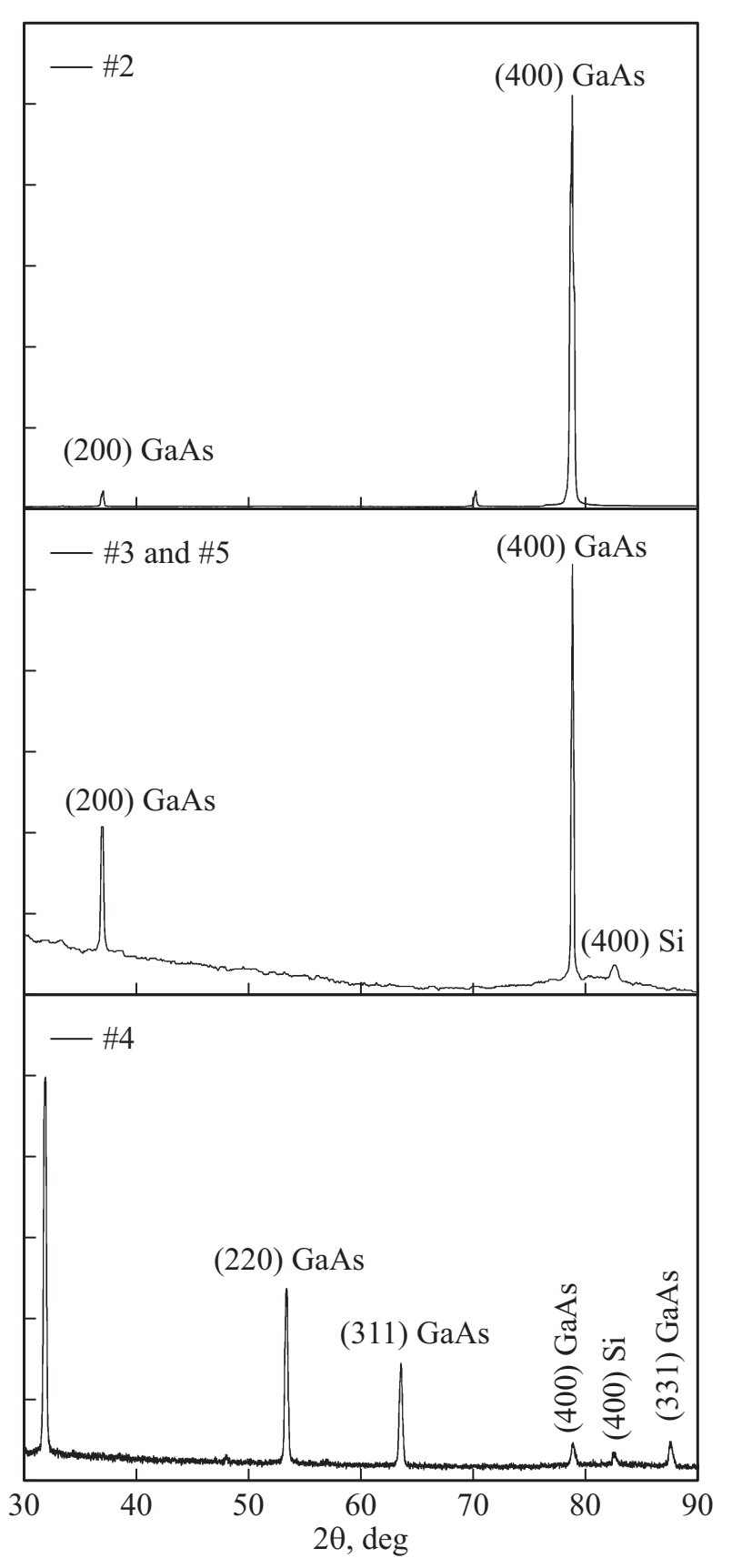

Рис. 2. Обзорные дифрактограммы эпитаксиальных гетероструктур, выращенных на $\operatorname{GaAs}(100)$ и подложках $\mathrm{Si}(100)$ с разной ориентацией.

присутствуют лишь дифракционные рефлексы (200), (400) характерные для дифракции для монокристаллического GaAs с ориентацией (100). Дифрактограммы образцов \#3 и \#5 совпадают, и на рисунке приведена лишь обзорная дифракция от образца \#3. Как видно из экспериментальных данных, помимо рефлексов (200) и (400) от слоя GaAs на дифрактограмме присутствует рефлекс (400) от подложки $\mathrm{Si}(100)$. Присутствие на дифрактограмме лишь рефлексов с отражениями (200) и (400) показывает монокристаллический рост слоя GaAs.
Что касается дифракции от образца \#4, то, как видно из полученных результатов, эпитаксиальная пленка имеет поликристаллическую структуру, так как на общей дифрактограмме присутствуют рефлексы, характерные для роста монокристаллической пленки в направлении (100). Следует также отметить, что исходя из полученных результатов рентгеноструктурного анализа хорошо видно наличие текстуры в направлении (111) у пленки образца \#4.

Поскольку образцы \#3 и \#4 имеют одинаковый тип подложки $-\mathrm{Si}(100)$ с разориентацией $\sim 3^{\circ}$ к [110], но отличаются лишь тем, что подложка $\mathrm{Si}$ у образца \#3 была предварительно протравлена перед процессом эпитаксии (сформирован переходной пористый нанослой), то из полученных результатов следует, что предварительное травление подложки $\mathrm{Si}(100)$ способствует монокристаллическому росту эпитаксиальной пленки $\mathrm{A}^{\mathrm{III}} \mathrm{B}^{\mathrm{V}}$ на кремнии даже при меньшей, чем обычно используется, разориентации подложки $[5,7]$. В то же время использование подложки с разориентацией $\sim 7.5^{\circ}$ к [110] не ведет к появлению поликристалличности в эпитаксиальной пленке без предварительной обработки подложки в виде травления.

Структурное качество образцов гетероструктур, выращенных на подложках $\mathrm{GaAs}$ и $\mathrm{Si}$ с различной степенью разориентации, было выполнено с применением высокоразрешающей рентгеновской дифракции. Такие исследования были проведены нами с использованием карт обратного $q$-пространства образцов, поскольку именно они позволяют получать прямую информацию о рассогласовании параметров кристаллических решеток у эпитаксиальной пленки и подложки, разориентации или релаксации слоя, плотности дислокаций в нем, его мозаичности или кривизне $[13,14]$.

Так на рис. 3 представлены карты распределения интенсивности дифрагированного излучения в $q$-пространстве вокруг симметричного узла (400) и асимметричного (511) для серии образцов \#2, \#3, \#5 с монокристаллическим эпитаксиальным слоем.

Анализ симметричного и асимметричного сканов обратного пространства показывает, что на картах образца \#2 присутствует лишь один узел, соответствующий отражению от GaAs, что указывает на когерентный рост эпитаксиальных пленок GaAs на подложках $\operatorname{GaAs}(100)$ и высокое структурное совершенство полученных слоев.

На картах образцов \#3 и \#5 помимо узла от подложки $\mathrm{Si}$ также присутствует узел, соответствующий отражению от GaAs. Анализ формы этого отражения свидетельствует об отсутствии в эпитаксиальной пленке GaAs антифазных доменов, возникновение которых, как уже было отмечено выше, обусловлено ростом полярного полупроводника на неполярной подложке.

Анализ карт обратного пространства образцов \#3 и \#5 показал, что положение и форма узлов эпитаксиального слоя GaAs в обратном пространстве, как для симметричного, так и для асимметричного отражения, изменяется 

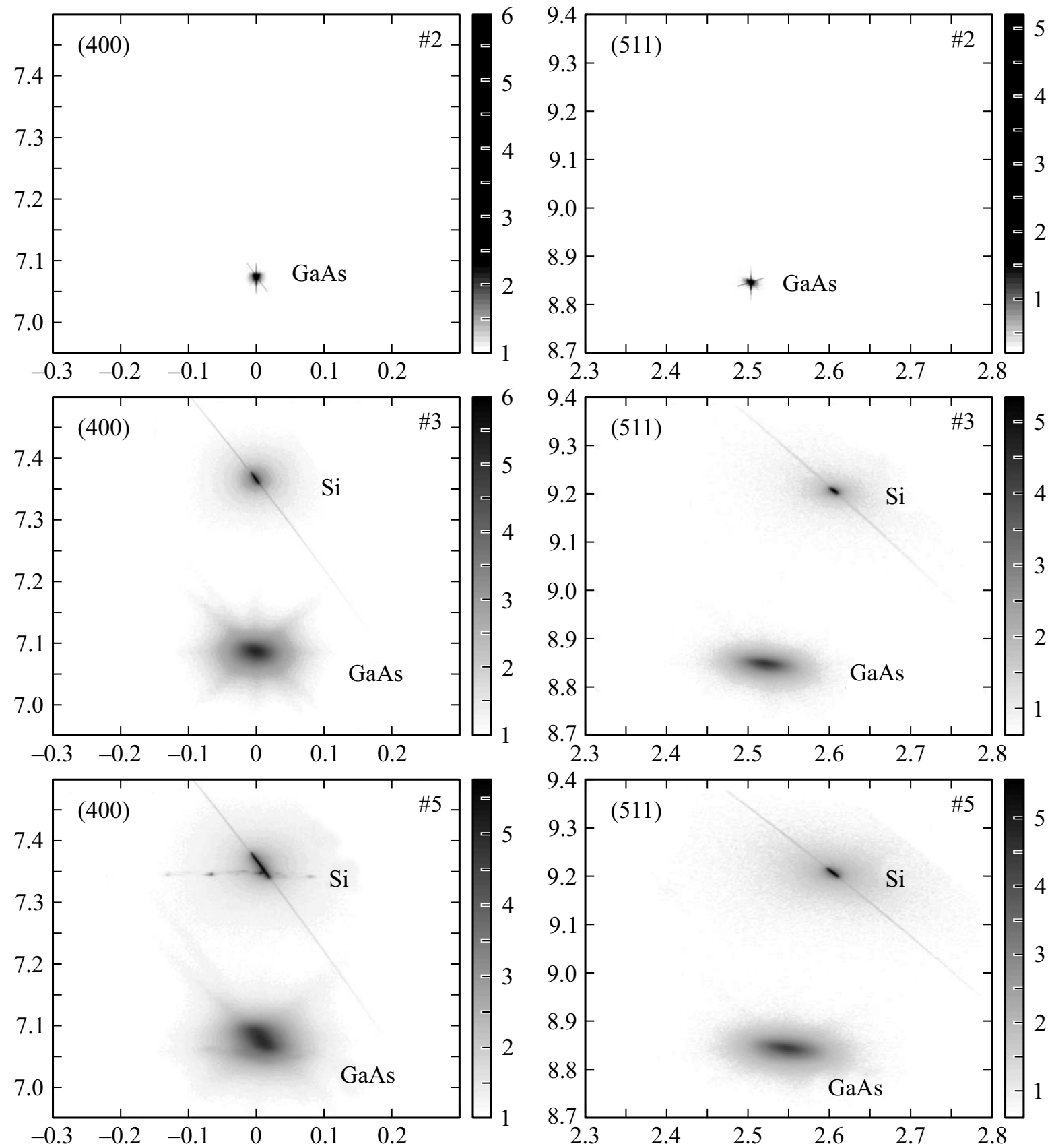

Рис. 3. Карты обратного $q$-пространства, полученные вокруг узлов (004) (слева) и (115) (справа) эпитаксиальных гетероструктур, выращенных на $\operatorname{GaAs}(100)$ и подложках $\operatorname{Si}(100)$ с разной ориентацией.

относительно его положения на картах образца \#2. Вид этих изменений не только свидетельствует об изменении параметра решетки эпитаксиального слоя GaAs по отношению к параметру подложки $\mathrm{GaAs}(100)$, но и свидетельствует об изменении симметрии кристаллической решетки пленки GaAs, с кубической на тетрагональную. Кроме того, хорошо заметно размазывание узлов на картах (511) в направлении, параллельном плоскости роста $q^{\|}$. Экспериментальные результаты показывают, что эпитаксиальный слой $\mathrm{GaAs}$, выросший на подложках $\mathrm{Si}$, находится в напряженном состоянии.
Кроме того, существующие изменения формы узлов GaAs на картах обратного пространства (характерные уширения узлов в направлении) свидетельствуют о появлении достаточно большого числа дефектов и градиенте напряжений в эпитаксиальном слое в направлении роста.

Следует также отметить появление необычной субструктуры в образце \#5, полученном на подложке кремния $\mathrm{Si}(100)$ с разориентацией $\sim 7.5^{\circ}$ к [110]. Из данных, приведенных на карте (004), видно появление дополнительных сателлитов, расположенных вдоль одной линии 
Таблица 2. Результаты высокоразрешающей рентгеновской дифрактометрии

\begin{tabular}{c|c|c|c|c|c|c|c}
\hline Образец & $a_{f}^{\perp}, \AA$ & $a_{f}^{\|}, \AA$ & $\mathrm{a} a_{\text {GaAs }}^{v}, \AA$ & $\varepsilon$ & $\varepsilon^{\|}$ & $\varepsilon^{\perp}$ & Степень релаксации, \% \\
\hline$\# 2$ & 5.6533 & 5.6532 & 5.6532 & - & - & - & 0 \\
$\# 3$ & 5.6553 & 5.6186 & 5.6378 & 0.0382 & 0.0347 & 0.042 & 6.9 \\
$\# 5$ & 5.6549 & 5.5526 & 5.6062 & 0.0324 & 0.0226 & 0.041 & 21.1
\end{tabular}

в плоскости роста вокруг узла Si. Вероятнее всего, этот факт является следствием образования серии тонких переходных слоев на кремнии с высоким уровнем напряжений в структуре (первоначальный ступенчатый рост из-за большой разориентации подложки).

Учитывая особенности метода высокоразрешающей рентгеновской дифракции, параметры кристаллической решетки в направлении роста $a^{\perp}$ и в плоскости роста $a^{\|}$ можно определить исходя из данных, полученных при анализе карт обратного $q$-пространства для симметричного (400) и асимметричного (511) рефлексов. Так, $a^{\perp}$ может быть определено на основе информации от симметричного (400) отражения как

$$
b_{400}^{\text {epilayer }}=\left(\frac{h^{2}}{a^{\perp}}\right)^{1 / 2}
$$

или асимметричного рефлекса

$$
b_{500}^{\text {epilayer }}=\left(\frac{h^{2}}{a^{\perp}}\right)^{1 / 2}
$$

в то время как $a^{\|}$задается следующим соотношением, учитывая данные лишь асимметричного (511) отражения:

$$
b_{001}^{\text {epilayer }}=\left(\frac{\left(k^{2}+l^{2}\right)^{2}}{a^{\|}}\right)^{1 / 2} .
$$

Здесь $b-$ координаты узлов в обратном $q$-пространстве.

Исходя из общей теории упругости и вводя коэффициент Пуассона для кубического кристалла $v$, параметр кристаллической решетки эпитаксиальной пленки с учетом внутренних напряжений, обозначаемый стандартно как $a^{v}$, в соответствии с линейной теорией упругости может быть рассчитан следующим образом $[15,16]$ :

$$
a_{\mathrm{GaAs}}^{v}=a_{\mathrm{GaAs}}^{\perp} \frac{1-v_{\mathrm{GaAs}}}{1+v_{\mathrm{GaAs}}}+a_{\mathrm{GaAs}}^{\|} \frac{2 v_{\mathrm{GaAs}}}{1+v_{\mathrm{GaAs}}} .
$$

Рассчитанные компоненты параметра решетки для эпитаксиального слоя GaAs и подложки $\mathrm{Si}$, а также параметр решетки GaAs с учетом внутренних напряжений, рассчитанный в соответствии с (4), представлены в табл. 2. Используя полученные данные о компонентах параметра кристаллической решетки, в общем случае мы можем определить относительные деформации, так как растяжения кристаллической решетки при наличии приложенной силы, которые индуцируют в пленке изменение решетки от кубической к тетрагональной, понижают симметрию кристалла. В простейшем случае мы можем различать параллельные $\Delta a^{\|} / a_{s}$ и перпендикулярные $\Delta a^{\perp} / a_{s}$ составляющие решетки и рассогласование на границе между пленкой и подложкой. Таким образом, перпендикулярная и параллельная деформации могут быть определены как [17]

$$
\varepsilon^{\|}=\frac{\Delta a^{\|}}{a_{s}}=\frac{a_{f}^{\|}-a_{s}}{a_{s}} \quad \text { и } \quad \varepsilon^{\perp}=\frac{\Delta a^{\perp}}{a_{s}}=\frac{a_{f}^{\perp}-a_{s}}{a_{s}},
$$

где $a_{f}^{\|}, a_{f}^{\perp}-$ параметры кристаллической решетки в плоскости и направлении роста эпитаксиальной пленки, $a_{s}$ - параметр решетки монокристаллической подложки.

На основе данных высокоразрешаюшей дифрактометрии можем рассчитать степень релаксации эпитаксиальной пленки GaAs [7]:

$$
\operatorname{Re} l a x=\frac{a_{\mathrm{GaAs}}^{v}-a_{s}}{a_{\mathrm{GaAs}}-a_{s}}
$$

где $a_{\mathrm{GaAs}}$ - параметр ненапряженного GaAs.

В табл. 2 представлены результаты расчета перпендикулярной и параллельной деформаций, общей деформации, а также степени релаксации в эпитаксиальном слое образцов.

\section{2. Микроскопические исследования}

Микроскопия сколов гетероструктур, выполненная с помощью сканирующего электронного микроскопа, позволила уточнить толщины слоев, а также оценить качество гетероинтерфейсов и наличие дефетов в пленках. На рис. 4, $а$ представлены микрофотографии скола гомоэпитаксиальной структуры \#2 в режиме отраженных электронов (рис. 4, $a$, слева) и режиме вторичных электронов (рис. 4, $a$, справа), а также микрофотография скола для образца \#3, полученная в режиме вторичных электронов (рис. 4,b). Анализ полученных экспериментальных результатов демонстрирует идеальное качество гетероинтерфейса и микроструктуры в эпитаксиальном слое GaAs, полученном на подложке $\mathrm{GaAs}(100)$ (образец \#2). В то же время для интегрированной гетероструктуры \#3, у которой эпитаксиальная пленка GaAs выращена на подложке $\mathrm{Si}(100)$ с разориентацией подложки $\sim 3^{\circ}$ к [110] и предварительным травлением, по данным сканирующей электронной микроскопии, наблюдается ярковыраженный гетеропереход, а также хорошо видны дислокации, прорастающие сквозь толщу пленки 


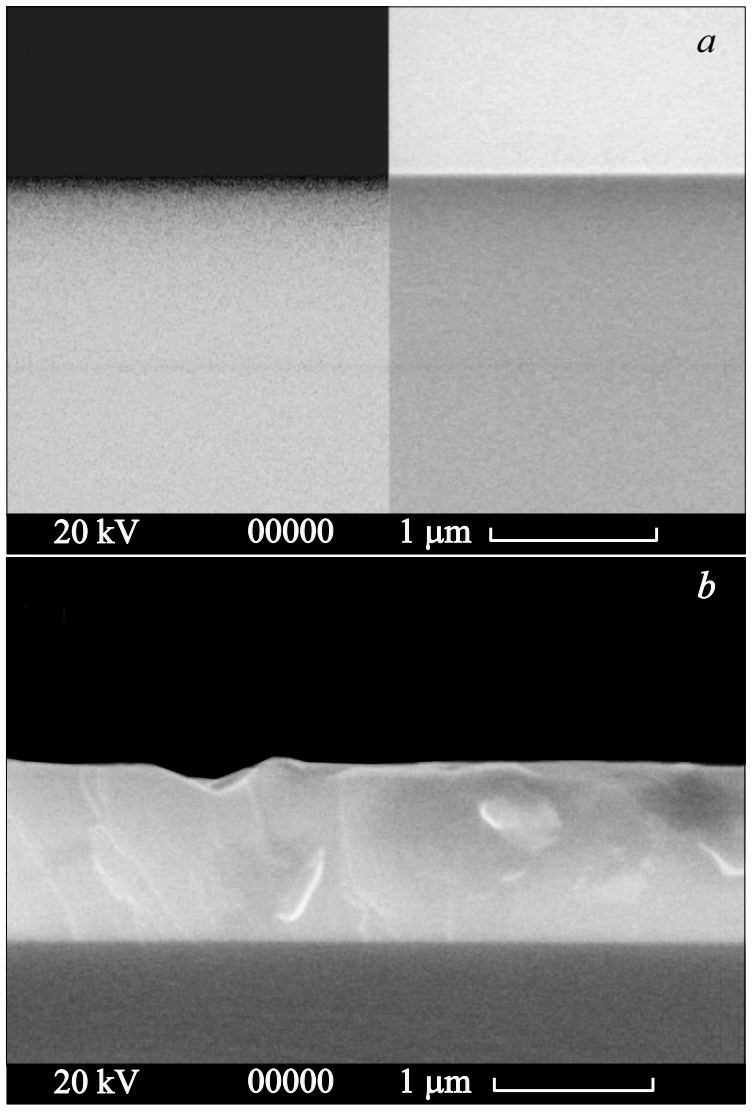

Рис. 4. Микрофотографии сколов гомоэпитаксиальной структуры \#2 в режиме отраженных электронов (рис. 4, $a$, слева) и в режиме вторичных электронов (рис. $4, a$, справа), а также микрофотография скола для интегрированной гетероструктуры \#3, полученная в режиме вторичных электронов (рис. $4, b$ ).

и объемные дефекты. Аналогичная картина наблюдается у образца $\# 5$ с разориентацией у подложки $\sim 7^{\circ}$.

\section{3. ИК-спектроскопия}

Многократно было показано [13,18], что метод ИК-спектроскопии весьма успешно используется при анализе тонких решеточных свойств и оценке структурного качества эпитаксиальных гетероструктур с тонкими слоями твердых растворов, выращенных на толстых монокристаллических подложках различных ориентаций при проведении съемки на отражение в далекой ИК-области спектра вблизи полосы остаточных лучей, с возникновением однофононного резонанса. Получение информации о фононном спектре является исключительно важным с точки зрения практических применений низкоразмерных структур в микро- и оптоэлектронных устройствах, поскольку фононы влияют на скорость релаксации возбужденных электронов и на подвижность носителей заряда.

На рис. 5 приведены ИК-спектры отражения от исследуемых эпитаксиальных структур. Как видно из

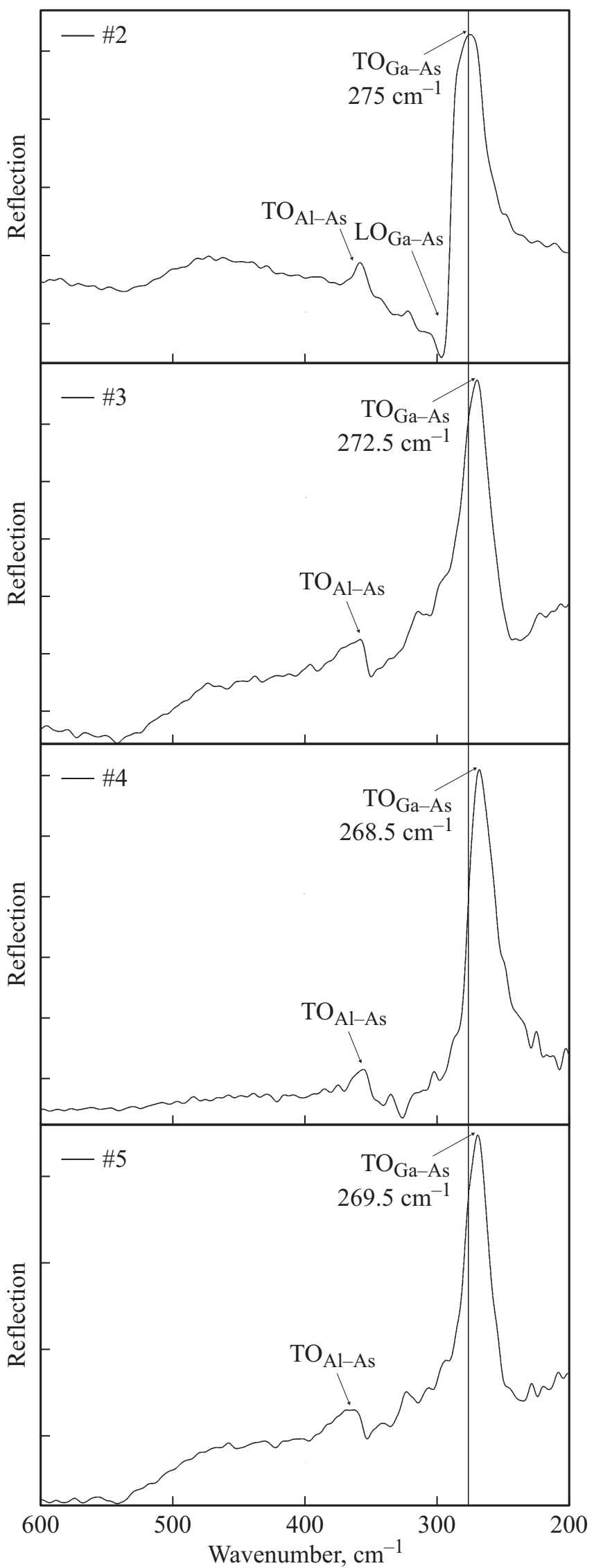

Рис. 5. Экспериментальные ИК-спектры отражения от исследуемых эпитаксиальных структур. 
эксперимента, в ИК-спектре отражения гетероструктуры \#2 присутствует высокоинтенсивная фононная мода $(\mathrm{TO}+\mathrm{LO})_{\mathrm{Ga}-\mathrm{As}}$ с формой, характерной для монокристаллического арсенида галлия, а также низкоинтенсивный поперечный оптический фонон $\mathrm{TO}_{\mathrm{Al}-\mathrm{As}}$ от верхнего cup-layer гетероструктуры. Частоты присутствующих в спектре продольных LO и поперечных ТО оптических фононов хорошо согласуются с уже существующими литературными данными $[19,20]$. Что касается ИК-спектров отражения интегрированных гетероструктур $\mathrm{GaAs} / \mathrm{Si}(100)$, то следует отметить, что в них также присутствуют фононные колебания $\mathrm{Ga}-\mathrm{As}$ и $\mathrm{Al}-\mathrm{Ga}$. При этом из эксперимента видно (см. рис. 5), что форма основной фононной моды меняется и для образцов \#3 и \#5 приобретает вид, характерный для образцов с плазмон-фононным резонансом [21]. Это в соответствии с результатами ряда работ [22-24], скоpeе всего, является следствием измененной концентрации носителей заряда в эпитаксиальной пленке GaAs, выращенной на подложке кремния с отклонением от сингулярного направления [100], по отношению к слою $\mathrm{GaAs}$, полученному на точно ориентированной подложке $\operatorname{GaAs}(100)$. Кроме того, хорошо заметно, что частота поперечной фононной моды $\mathrm{TO}_{\mathrm{Ga}-\mathrm{As}}$ для образцов \#3-\#5 смещена в низкочастотную область относительно ее положения в спектре гомоэпитаксиального образца \#2, что подтверждает факт возникновения внутренних напряжений кристаллической решетки в эпитаксиальном слое. Величина сдвига частоты поперечного оптического фонона $\mathrm{TO}_{\mathrm{Ga}-\mathrm{As}}$ в образцах интегрированных гетероструктр $\mathrm{GaAs} / \mathrm{Si}(100)$ прямо пропорциональна величине коэффициента релаксации эпитаксиальной пленки GaAs.

\section{4. ФЛ-спектроскопия}

Структурное качество эпитаксиальных пленок GaAs, а также изменение стехиометрии в эпитаксиальном слое при росте на подложках $\mathrm{Si}$ неизменно должны отразиться на характере и величине квантового выхода фотолюминесценции от образца, т.е. на энергетических характеристиках арсенида галлия. Для проверки этого утверждения нами были получены спектры фотолюминесценции образцов при комнатной температуре. ФЛ-спектры образцов приведены на рис. 6 без нормировки.

Из полученных данных хорошо видно, что в спектре образца \#2, выращенного ГФЭ на подложке $\operatorname{GaAs}(100)$, присутствует лишь один максимум с энергией $\sim 1.42$ эВ и представляющий собой переход зона-зона (эмиссию от гомоэпитаксиального слоя). Что же касается эмиссии от образцов \#3 и \#5, то, как видно из рис. 6 , в спектрах этих структур присутствует также один максимум люминесценции, который сдвинут в область больших энергий (меньших длин волн) и локализован в области $\sim 1.48$ эВ. Следует отметить, что полуширина полосы фотолюминесценции у образцов \#3 и \#5 ( $\triangle \# 3 \sim 19$ нм, $\Delta \# 5 \sim 21$ нм) незначительно выше полуширины пика

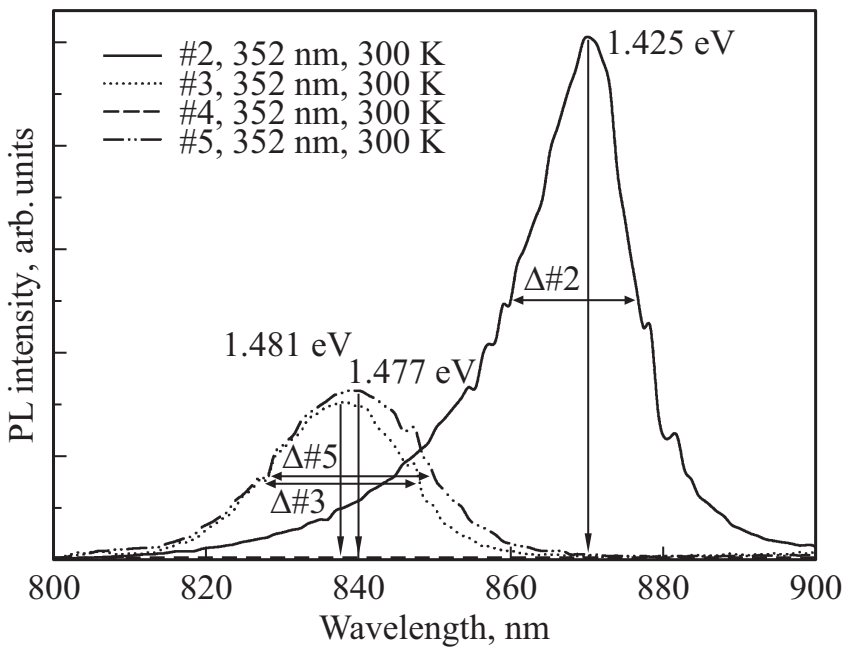

Рис. 6. Спектры фотолюминесценции гомоэпитаксиальной структуры $\mathrm{GaAs} / \mathrm{GaAs}(100)$ и интегрированных гетроструктур $\mathrm{GaAs} / \mathrm{Si}(100)$, полученные при комнатной температуре.

фотолюминесценции гомоэпитаксиального образца \#2 $(\Delta \# 2 \sim 16 \mathrm{Hм})$. Что же касается энергетического сдвига полосы фотолюминесценции в спектрах образцов, то, на наш взгляд, сдвиг обусловлен возникновением внутренних напряжений решетки в эпитаксиальном слое из-за рассогласования параметров кристаллической решетки у эпитаксиального слоя и подложки. Кроме того, интенсивность фотолюминесценции интегрированных образцов $\mathrm{GaAs} / \mathrm{Si}(100)$ более чем в 2 раза ниже, чем эмиссия от слоя GaAs, выращенного на подложке $\mathrm{GaAs}$, что является следствием наличия дефектов в эпитаксиальном слое. Эмиссия от образца \#4, полученного на подложке $\mathrm{Si}(100)$ с разориентацией $\sim 3^{\circ}$ к [110] без травления, практически отсутствует (имеет весьма низкую интенсивность), что подтверждает факт поликристаллического состояния пленки GaAs.

\section{5. УФ-спектроскопия}

Для изучения оптических свойств в УФ-диапазоне интегрированных эпитаксиальных гетерострутур $\mathrm{GaAs} / \mathrm{Si}(100)$ мы использовали подход с применением методики съемки на отражение, апробированный нами в серии предыдущих работ [25-28]. Предлагаемая методика позволяет получить информацию об оптических свойствах в УФ-диапазоне пленок/слоев, нанесенных/сформированных на объемных подложках. Спектры пропускания-отражения интегрированных гетероструктур были получены при угле падения электромагнитного излучения, близком к нормали, в области длин волн $190-900$ нм. С целью выявления механизмов оптического поглощения в слоях, сформированных методом электрохимического травления с использованием возможностей программы OPUS Bruker и с учетом формулы Ламберта-Бугера: $T=\exp [-D]$, где $T-$ пропускание, 
Таблица 3. Результаты анализа зависимостей $(D \cdot h v)^{2}$ образцов гетероструктур

\begin{tabular}{|c|c|c|c|c|c|}
\hline \multirow{2}{*}{ Образец } & \multicolumn{5}{|c|}{$\begin{array}{c}\text { Прямые переходы и особенности в УФ спектрах, а также } \\
\text { их принадлежность в соответствии с данными из работы [30] }\end{array}$} \\
\hline & $\Gamma 15-\Gamma 1$, эВ & $L 3-L 1, \ni \mathrm{B}$ & $\Lambda 3-\Lambda 1$, эB & $\begin{array}{c}\Gamma 15-Г 15 \\
\text { (область } a), \text { эВ }\end{array}$ & $X 5-X 1$, эВ \\
\hline$\# 2$ & 1.15 & 2.55 & & 4.21 & 4.87 \\
\hline \#3 & 1.25 & 2.15 & 4.38 & 4.53 & \\
\hline \#4 & 1.48 & - & 3.42 & - & - \\
\hline$\# 5$ & 1.60 & - & 3.65 & - & - \\
\hline
\end{tabular}

$D$ - оптическая плотность мы перестроили спектры пропускания-отражения в спектры поглощения.

На рис. 7 представлены зависимости $(D \cdot h v)^{2}$ от энергии квантов для образцов гетероструктур, которые были рассчитаны из спектра зеркального отражения с использованием соотношений Крамерса-Кронига [29].

Графический анализ зависимостей $(D \cdot h v)^{2}$ от энергии квантов позволил выявить отдельные участки с линейной зависимостью $(D \cdot h v)^{2}$ от энергии квантов, что может указывать на наличие в этой спектральной области прямых разрешенных переходов (рис. 7). Линейная экстраполяция данных участков к нулевому значению позволяет определить энергию прямых переходов, характерных для образцов.

Максимумы в УФ-спектрах арсенида галлия соответствуют переходам в точках $\Gamma, L$ и $\Lambda$, среди которых переходы в точках $L$ и $\Lambda$ являются дублетами в результате спин-орбитального расщепления валентной зоны.

В табл. 3 приведены энергии переходов и особенности в спектрах для исследованных в работе образцов, определенные по вышеописанной методике. Следует отметить, что определенные нами в результате расчетов энергии прямых переходов из валентной зоны в зону проводимости для образца \#2, представляющего собой гомоэпитаксиальную структуру $\mathrm{GaAs} / \mathrm{GaAs}(100)$, имеют сдвиг от литературных данных, однако он не превышает 0.3 эВ. Этот сдвиг имеет место ввиду того, что зависимости $(D \cdot h v)^{2}$ от энергии квантов были построены не из прямых данных (спектров пропускания/поглощения пленок), а были рассчитаны с использованием результатов пересчета спектров пропускания-отражения в спектры поглощения.

Что же касается особенностей в спектрах интегрированных гетероструктур $\mathrm{GaAs} / \mathrm{Si}(100)$, то следует отметить, что лишь в спектре образца \#3, выращенного на подложке $\mathrm{Si}$ с разориентацией $\sim 3^{\circ}$ к [110] и предварительным ее травлением, в котором эпитаксиальный слой $\mathrm{GaAs}$ имеет наименьший коэффициент релаксации кристаллической решетки, присутствуют аналогичные спектру гомоэпитаксиальной структуры \#2 переходы и
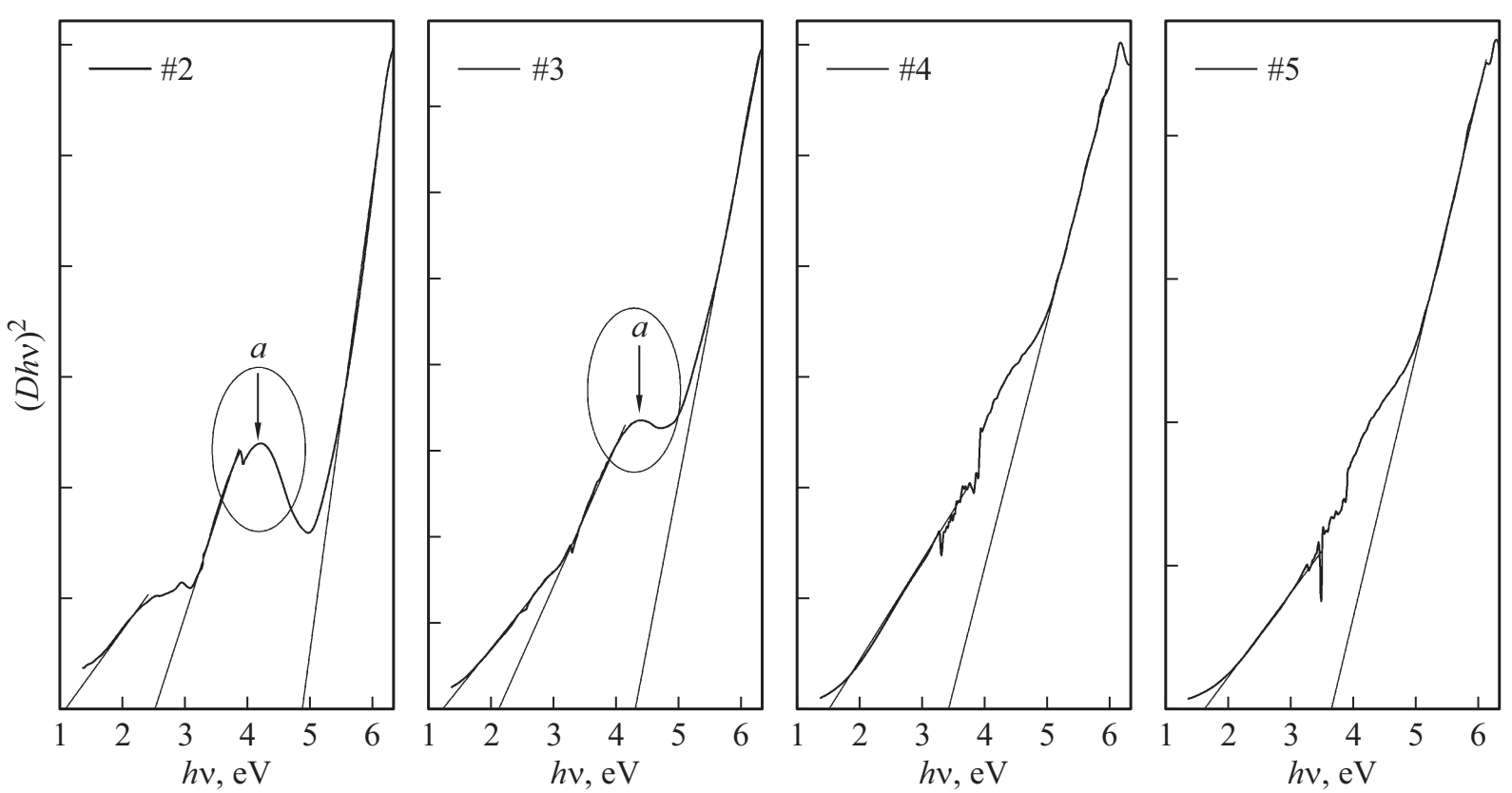

Рис. 7. Зависимости $(D \cdot h v)^{2}$ от энергии квантов для образцов гетероструктур. 
особенности, в том числе в области $A$ на спектре (см. рис. 7). В спектрах образцов \#4 и \#5 присутствуют лишь прямые переходы $\Gamma 15-\Gamma 1$ и $\Lambda 3-\Lambda 1$. Необходимо отметить, что с ростом коэффициента релаксации эпитаксиального слоя GaAs (см. табл. 2 и 3) отмечается сдвиг в сторону бо́льших энергий для разрешенных прямых переходов в точках Г и $\Lambda$, при этом для переходов в точках $L$ и $X$ характерен сдвиг в низкоэнергетическую область.

\section{4. Заключение}

С использованием метода химического осаждения из паровой фазы металлоорганических соединений (MOCVD) на подложках $\mathrm{Si}(100)$ с различной степенью разориентации от направления [100] и предварительной обработки нами были получены интегрированные эпитаксиальные гетероструктуры $\mathrm{GaAs} / \mathrm{Si}(100)$, структурные и оптические свойства которых были сопоставлены со свойствами GaAs, выращенного на точно ориентированной подложке $\mathrm{GaAs}(100)$. С привлечением комплекса структурно-спектроскопиченских методов анализа (высокоразрешающая рентгеновская дифракция с картированием обратного пространства, сканирующая электронная микроскопия, ИК-, ФЛ- и УФ-спектроскопия) были получены следующие результаты. Показано, что рост эпитаксиального слоя GaAs методом MOCVD при выбранных условиях роста на подложке $\mathrm{Si}$ с разориентацией $\sim 3^{\circ}$ приводит к поликристаллическому состоянию GaAs. Однако выполненная предварительная обработка этого типа подложки в виде травления, равно как и использование подложек с большей степенью разориентации $\sim 7^{\circ}$, позволяет получить пленку GaAs в монокристаллическом состоянии и без присутствия в ней антифазных доменов. Стоит отметить, что увеличение степени разориентации подложки с 3 до $7^{\circ}$ ведет к росту более чем в 3 раза коэффициента релаксации, что негативно отражается на ее структурном качестве. Эти данные находятся в хорошем согласовании с результатами ИК-спектроскопии на отражение.

Что же касается оптических свойств эпитаксиального слоя GaAs, полученного на подложках $\mathrm{Si}(100)$, то, как показали результаты ФЛ-спектроскопии, в спектрах образцов \#3 и \#5 так же, как и в спектре гомоэпитаксиального образца \#2, присутствует один максимум люминесценции. Энергетическое положение максимума в спектре ФЛ, полуширина полосы фотолюминесценции и ее интенсивность зависят от величины напряжений в эпитаксиальном слое GaAs и степени его релаксации. Следует отметить, что интенсивность фотолюминесценции у образцов интегрированных $\mathrm{GaAs} / \mathrm{Si}(100)$-структур более чем в 2 раза ниже, чем эмиссия от гомоэпитаксиального образца $\mathrm{GaAs} / \mathrm{GaAs}(100)$.

Особенности оптических свойств интегрированных гетероструктур $\mathrm{GaAs} / \mathrm{Si}(100)$ в УФ-области спектра также определяются значением коэффициента релаксации.
Из эксперимента следует, что лишь спектр образца, выращенного на подложке $\mathrm{Si}$ с разориентацией $\sim 3^{\circ}$ к [110] и предварительным травлением, максимально близок спектру гомоэпитаксиальной структуры, и в нем присутствуют все аналогичные переходы и особенности. Отмечено, что с ростом коэффициента релаксации эпитаксиального слоя GaAs сдвигаются энергии для разрешенных прямых переходов.

Таким образом, впервые удалось показать, что управление структурными и оптическими функциональными характеристиками интегрированных гетроструктур $\mathrm{GaAs} / \mathrm{Si}(100)$ может быть достигнуто за счет использования разориентированных подложек GaAs c их предварительным травлением. Рост эпитаксиального слоя GaAs на подложках кремния без образования антифазных доменов может быть выполнен на подложке отклоненной от сингулярной плоскости (001) менее чем на $4-6^{\circ}$, что в основном практикуется $[5,7]$, или без использования переходного слоя из наностолбиков GaAs [8].

Выполненная предварительная обработка подложки в виде травления позволяет получить методом ГФЭ эпитаксиальную пленку GaAs в монокристаллическом состоянии, без присутствия в ней антифазных доменов и значительно меньшим коэффициентом релаксации, что положительно отражается на ее структурном качестве. Эти данные находятся в хорошем согласовании с результатами ИК-спектроскопии на отражение, ФЛ- и УФ-спектроскопии.

Работа выполнена при поддержке гранта президента РФ МД-188.2017.2. В части технологии получения гетероструктур работа выполнена в соответствии с Государственным заданием ФТИ им. А.Ф. Иоффе. В части диагностики интегрированных структур работа поддержана грантом № 11.4718.2017/8.9 Министерства образования и науки России в рамках государственного задания вузам в сфере научной деятельности на 2017-2019 гг.

Авторы благодарны В.Б. Шуман за полезные советы по работе с кремниевыми пластинами.

\section{Список литературы}

[1] I. Prieto, R. Kozak, O. Skibitzki, M.D. Rossell, P. Zaumseil, G. Capellini. Nanotechnology, 28, 135701 (2017).

[2] Y. Shi, H. Guo, H. Ni, C. Xue, Z. Niu, J. Tang. Materials, 5, 2917 (2012).

[3] C-P. Chu, S. Arafin, T. Nie, K. Yao, X. Kou, L. He. Cryst. Growth Des., 14, 593 (2014).

[4] G. Deligeorgis, S. Gallis, M. Androulidaki, D. Cengher, Z. Hatzopoulos, M. Alexe, V. Dragoi, E.D. Kyriakis-Bitzaros, G. Hakias, F. Peiro, A. Georgakis. 12th Int. Conf. on Semiconducting and Insulating Materials (June 30-July 5 2002, Bratislava, Slovak Republic) p. 125.

[5] Y.B. Bolkhovityanov, O.P. Pchelyakov. Phys. Uspekhi, 51, 437 (2008).

[6] C. Renard, T. Moliére, N. Cherkashin, J. Alvarez, L. Vincent, A. Jaffré. Sci. Rep., 6, 25328 (2016). 
[7] C.S. Wong, N.S. Bennett, B. Galiana, P. Tejedor, M. Benedicto, J.M. Molina-Aldareguia. Semicond. Sci. Technol., 27, 115012 (2012).

[8] Q. Li, K.W. Ng, K.M. Lau. Appl. Phys. Lett., 106 (7), 072105 (2015).

[9] I. Prieto, R. Kozak, O. Skibitzki, M.D. Rossell, T. Schroeder, R. Erni. Small, 13, 1603122 (2017).

[10] Y. Buzynin, V. Shengurov, B. Zvonkov, A. Buzynin, S. Denisov, N. Baidus, M. Drozdov, D. Pavlov, P. Yunin. AIP Advances, 7, 015304 (1017).

[11] A.S. Brown. J. Vac. Sci. Technol. B, 16, 2308 (1998).

[12] K. Vanhollebeke, I. Moerman, P. Van Daele, P. Demeester. Prog. Cryst. Growth. Charact. Mater., 41, 1 (2000).

[13] P.V. Seredin, A.V. Glotov, A.S. Lenshin, I.N. Arsentyev, D.A. Vinokurov, T. Prutskij. Semiconductors, 48, 21 (2014).

[14] P.V. Seredin, V.E. Ternovaya, A.V. Glotov, A.S. Len'shin, I.N. Arsent'ev, D.A. Vinokurov. Phys. Sol. St., 55, 2161 (2013).

[15] E.P. Domashevskaya, P.V. Seredin, E.A. Dolgopolova, I.E. Zanin, I.N. Arsent'ev, D.A. Vinokurov. Semiconductors, 39, 336 (2005).

[16] P.V. Seredin, A.V. Glotov, E.P. Domashevskaya, I.N. Arsentyev, D.A. Vinokurov, I.S. Tarasov. Appl. Surf. Sci., 267, 181 (2013).

[17] P.V. Seredin, A.V. Glotov, E.P. Domashevskaya, I.N. Arsentyev, D.A. Vinokurov, A.L. Stankevich, I.S. Tarasov. Semiconductors, 44 (8), 1106 (2010).

[18] P.V. Seredin, A.V. Glotov, E.P. Domashevskaya, I.N. Arsentyev, D.A. Vinokurov, I.S. Tarasov. Phys. B: Condens. Matter, 405, 4607 (2010).

[19] J.O. Akinlami, A.O. Ashamu. J. Semicond., 34, 032002 (2013).

[20] Paul M. Amirtharaj, David G. Seiler. Optical Properties of Semiconductors. Handb. Opt. Vol. II. Devices Meas. Prop. Second Ed. 2nd ed. McGraw-Hill (1995).

[21] P.V. Seredin, A.V. Glotov, E.P. Domashevskaya, I.N. Arsentyev, D.A. Vinokurov, I.S. Tarasov. Semiconductors, 44, 184 (2010).

[22] K. Mochizuki, S. Goto, H. Kakibayashi, C. Kusano. Jpn. J. Appl. Phys., 29, L1046 (1990).

[23] P.R. Hageman, J. te Nijenhuis, M.J. Anders, L.J. Giling. J. Cryst. Growth, 170, 270 (1997).

[24] S. Orsila, A. Tukiainen, P. Uusimaa, J. Dekker, T. Leinonen, M. Pessa. J. Cryst. Growth, 227-228, 249 (2001).

[25] V.A. Volodin, M.D. Efremov, V.Y. Prints, V.V. Preobrazhenskii, B.R. Semyagin, A.O. Govorov. J. Exp. Theor. Phys. Lett., 66, 47 (1997).

[26] P.V. Seredin, A.S. Lenshin, V.M. Kashkarov, A.N. Lukin, I.N. Arsentiev, A.D. Bondarev. Mater. Sci Semicond. Process., 39, 551 (2015).

[27] P.V. Seredin, V.M. Kashkarov, I.N. Arsentyev, A.D. Bondarev, I.S. Tarasov. Phys. B: Condens. Matter, 495, 54 (2016).

[28] P.V. Seredin, A.S. Lenshin, D.L. Goloshchapov, A.N. Lukin, I.N. Arsentyev, A.D. Bondarev. Semiconductors, 49, 915 (2015).

[29] Y.I. Ukhanov. Optical properties of semiconductors (M. Nauka, 1977).

[30] J. Tauc. Optical properties of semiconductors in the visible and ultra-volet ranges. Progress in Semiconductors (Heywood, London, 1965).

\section{Influence of misorientation of the substrate and its preliminary etching on the structural and optical properties of integrated GaAs/Si(100) MOCVD heterostructures}

P.V. Seredin ${ }^{1}$, D.L. Goloshchapov' ${ }^{1}$, D.S. Zolotukhin ${ }^{1}$ A.S. Lenshin ${ }^{1}$, A.N. Lukin ${ }^{1}$, Yu.Yu,. Khudyakov' ${ }^{1}$, I.N. Arsentyev ${ }^{2}$, A.V. Zhabotinsky ${ }^{2}$, D.N. Nikolaev ${ }^{2}$, N.A. Pikhtin ${ }^{2}$

\author{
${ }^{2}$ Voronezh State University, \\ 394006 Voronezh, Russia \\ ${ }^{2}$ loffe Institute, \\ 194021 St. Petersburg, Russia
}

\begin{abstract}
We report on the successful control of optical and functional properties of the $\mathrm{GaAs} / \mathrm{Si}(100)$ heterostructures due to the use of the pre-etched vicinal $\mathrm{Si}(100)$ substrates. The usage of vicinal $\mathrm{Si}(100)$ substrates with low off-angle $4-6^{\circ}$ toward (001) plane or absence of nanoculumnar GaAs buffer layer helps to achieve thick GaAs layer without anti-phase domain incorporations formation by MOCVD technique. XRD data analysis showed that growth on pre-etched $\mathrm{Si}(100)$ substrates results in monocrystalline layer formation with lower strain relaxation retio compared to un-etched $\mathrm{Si}(100)$ substrate. Low relaxation ratio is positively affects on GaAs structural quality since as the main way of stress relexation is threading dislocations formation. XRD data are correlates well with IR, UV and PL spectra as optical properties are directly related with heterostructure strain relaxation ratio.
\end{abstract}

